EGU2020-17868

https://doi.org/10.5194/egusphere-egu2020-17868

EGU General Assembly 2020

(c) Author(s) 2021. This work is distributed under

the Creative Commons Attribution 4.0 License.

\title{
On the accuracy of gravity fields obtained with Newton integrals on a hollow sphere
}

\author{
Ludovic Jeanniot ${ }^{1}$, Cedric Thieulot ${ }^{1}$, Bart Root ${ }^{2}$, John Naliboff ${ }^{3}$, and Wim Spakman ${ }^{1}$ \\ ${ }^{1}$ Utrecht University, Faculty of Geosciences, Earth Sciences Mantle Dynamics, Utrecht, Netherlands (I.jeanniot@uu.nl) \\ ${ }^{2}$ Delft University of Technology, Delft, Netherlands (B.C.Root@tudelft.nl) \\ ${ }^{3}$ New Mexico Tech University, New Mexico, US
}

The mass-density distribution of the Earth drives mantle convection and plate tectonics but is poorly known. We aim to predict gravity fields as a constraint for geodynamical modelling. In order to compute synthetic Earth gravity one must define a spherical geometry filled with a density model. Density models for the whole mantle down to the CMB come from tomographic models which therefore require converting speed waves velocities to density using a scaling factor.

We use a discretised integration method to compute globally gravity acceleration, gravity anomalies, potential and gradients, in the state of the art finite element code ASPECT.

Three density models are tested separately: a density field obtained from SL2013 and S40RTS tomographic models for the deep mantle, and the density model CRUST1.0 for the thin upper lithosphere layer. We combine these 3 datasets into one to create a composite model which is compared to the global seismic model LLNL-G3D-JPS of Simmons et al. (2015). We test the sensitivity of gravity prediction on the use of various conversion scaling factors of shear wave velocity to density. We find that the scaling factor profile also has a major impact on gravity prediction.

Finally, we present early results of the gravity field prediction for two local areas, the Indian-Tibet plate boundary and the Mediterranean Sea. Gravity predictions are compared to satellite gravity. 